\title{
Photovoltaic System Performance Enhancement With Non-Tracking Planar Concentrators: Experimental Results and Bi-Directional Reflectance Function (BDRF) Based Modelling
}

\author{
Rob W. Andrews *, Andrew Pollard* and Joshua M. Pearce ${ }^{\dagger}$ \\ * Department of Mechanical and Materials Engineering \\ Queen's University, Kingston, Ontario, Canada \\ $\dagger$ Department of Materials Science and Engineering and the Department of Electrical and Computer Engineering \\ Michigan Technological University, Houghton, MI, USA
}

\begin{abstract}
Non-tracking planar concentrators are a low-cost method of increasing the performance of traditional solar photovoltaic (PV) systems. This paper presents new methodologies for properly modeling this type of system design and experimental results using a bi-directional reflectance function (BDRF) of non-ideal surfaces rather than traditional geometric optics. This methodology allows for the evaluation and optimization of specular and non-specular reflectors in planar concentration systems. In addition, an outdoor system has been shown to improve energy yield by $45 \%$ for a traditional flat glass module and by $40 \%$ for a prismatic glass crystalline silicon module when compared to a control module at the same orientation. When compared to a control module set at the optimal tilt angle for this region, the energy improvement is $18 \%$ for both system. Simulations show that a maximum increase of $30 \%$ is achievable for an optimized system located in Kingston, $O N$ using a reflector with specular reflection and an integrated hemispherical reflectance of $80 \%$. This validated model can be used to optimize reflector topology to identify the potential for increased energy harvest from both existing PV and new-build PV assets.
\end{abstract}

Index Terms-planar concentrator, low concentration, crystal silicon,optics, BDRF, reflectors, booster mirrors, photovoltaic, photovoltaic system

\section{NOMENCLATURE}

\begin{tabular}{|c|c|}
\hline$G_{\text {diff }}$ & Diffuse irradiation $\left(W / m^{2}\right)$ \\
\hline$G_{D N I}$ & Direct normal irradiation $\left(W / m^{2}\right)$ \\
\hline$G_{p}$ & $\begin{array}{l}\text { DNI projected onto the vertical plane } \\
\left(W / m^{2}\right)\end{array}$ \\
\hline$G_{m}^{i}$ & $\begin{array}{l}\text { Irradiance on the surface of the module due } \\
\text { to reflection }\left(\mathrm{W} / \mathrm{m}^{2}\right)\end{array}$ \\
\hline$E_{r}^{i}$ & Energy incident on the reflector surface $(W)$ \\
\hline$E_{r}^{o}$ & $\begin{array}{l}\text { Energy leaving the reflector surface, per } \\
\text { degree }(W / \theta)\end{array}$ \\
\hline$E_{m}^{i}$ & $\begin{array}{l}\text { Energy incident on the surface of the mod- } \\
\text { ule }(W)\end{array}$ \\
\hline$T_{a}$ & Ambient temperature $(C)$ \\
\hline$A_{m}$ & $\begin{array}{l}\text { Module Area assuming a unit depth } \\
\left(\int d z=1\right)\left(m^{2}\right)\end{array}$ \\
\hline$A R$ & $\begin{array}{l}\text { Area ratio of illuminated to non-illuminated } \\
\text { module area }\end{array}$ \\
\hline$F_{r m}$ & View factor between module and reflector \\
\hline$x$ & Dimension along the module $(m)$ \\
\hline$y$ & long the reflector $(m)$ \\
\hline$y$ & Dimension orthogonal to $x$ and $y(m)$ \\
\hline & Direct distance between $d x$ and $d y(m)$ \\
\hline wrefl & Reflector length $(m)$ \\
\hline
\end{tabular}

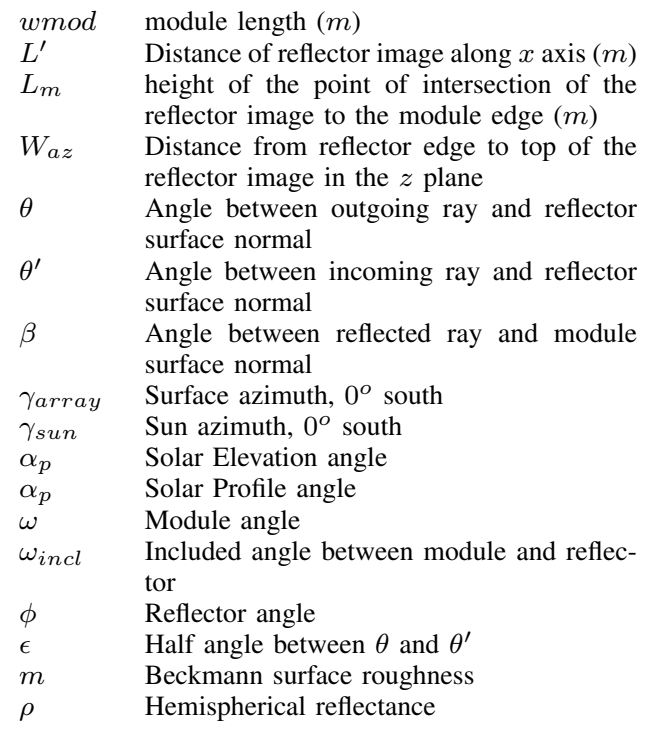

\section{INTRODUCTION}

Solar photovoltaic (PV) systems are a rapidly expanding sustainable renewable energy market, and will play a large role in the future sustainable energy mix [1], [2]. Currently, commercial and utility scale PV installations are predominately arranged in multiple parallel rows of flat modules, which are aligned towards the mean maximum solar intensity [3].

Modules must be installed with a setback from the row in front of it, to reduce inter-row shading losses and allow maintenance access. The spacing of these rows is highly dependent on the latitude of the solar array, land availability, and economic constraints which determine the economic performance of a system [4]. Typically, the row spacing is designed to reduce inter-row shading losses that occur in the early morning and late afternoon; however, this arrangement leaves the spaces between rows illuminated during the periods of highest solar resource which is around solar noon [5].

Previous work has investigated the application of planar reflectors for both solar thermal and photovoltaic applications. Much of the early work on planar concentration was focused on the improvement of winter time yields for solar thermal systems [6]-[9]. Some studies found that the optimal orientation for this at high latitudes was a vertical collector with a horizontal reflector [8]. A large body of literature has 
also looked at various ray tracing models for estimating the increase in irradiation from a given reflector geometry [8] [18]. Of these models, there are some that account for diffuse reflectors that utilize a combined view factor and specular reflectance model [11], [13], [14], some that analyze a two dimensional specular reflectance model [18], and some that include experimental results [7], [10], [18], [119]

Currently, planar reflectors are utilized in district heating solar thermal plants in Sweden and Denmark [5], and have been shown experimentally to increase the thermal energy collection at sites of $60^{\circ} \mathrm{N}$ latitudes by around 30\% [18] compared to a module at the same tilt angle of $45^{\circ}$. It has been proposed that the introduction of non-specular corrugated booster reflectors may further increase the outputs of these fields by up to $8 \%$ [5] and the Bi Directional Reflectance Function (BDRF) of these corrugated materials also have been characterized [12].

There are limited examples of experimental studies that address the specific effects of low-level concentration on PV system losses, and recently a study was undertaken to identify these additional loss mechanisms [20].

Unfortunately, though the technical feasibility of these systems has been shown, at the time of publication only one entity has commercialized a comparable system for PV applications [21], and because of assumptions made in the design of the system, specialized parallel modules must be utilized with this system, which tend to increase the costs of the system.

In order to investigate the potential implementation of nontracking planar concentration PV systems for conventional low-cost PV modules, this study: 1) develops and validates a model using the concept of the BDRF of non ideal surfaces rather than geometric optics and 2) experimentally investigates systems in an outdoor test site in Kingston, Ontario utilizing commercially available crystalline silicon PV modules. The proposed model integrates previous work which analytically investigated the module temperature increases, diode loading, and angle of incidence effects. The outputs of this model are used to perform a sensitivity analysis which identifies important factors in the design of low-level concentration systems.

This chapter is derived from a technical paper submitted to the IEEE Photovoltaics Specialists Conference (PVSC) of the same name [22] study expands on this work through: 1) refining and further validating a model using the concept of the BDRF of non ideal surfaces rather than geometric optics, 2) undertaking laboratory scatterometry and solar simulator testing to compliment experimental data collected at an outdoor test site in Kingston, Ontario 3) utilizing the validated model to perform a sensitivity analysis which identifies important factors in the design of low-level concentration systems. It should be noted that the original reported boost of $35 \%$ for a prismatic glass module was erroneous, and upon further analysis it was found that the boost is in-fact $40 \%$

\section{EXPERIMENTAL APPARATUS}

\section{A. Outdoors testing}

A $6 \mathrm{~m} \times 2.5 \mathrm{~m}$ planar concentrator was installed at the Open Solar Outdoors Test Field (OSOTF) in Kingston, Ontario (44 $140 \mathrm{~N}, 76^{\circ} 300 \mathrm{~W}$ ) in the fall of 2011 [23]. Two stacks of landscape crystalline silicon (c-Si) PV modules were arranged in front of the wide planar reflector, and their actual locations

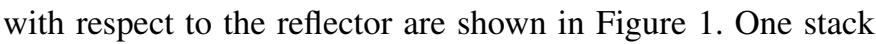
had modules with a prismatic glass front sheet, and the other stack had traditional flat glass modules. Each module had a length of $1 \mathrm{~m}$ in the plane shown in Figure $\square$ and the reflector had a length of $2.5 \mathrm{~m}$ in the same plane. Figure $\square$ shows the two dimensional domain used for the analysis and modeling of the system, and is described in detail in section III-A.

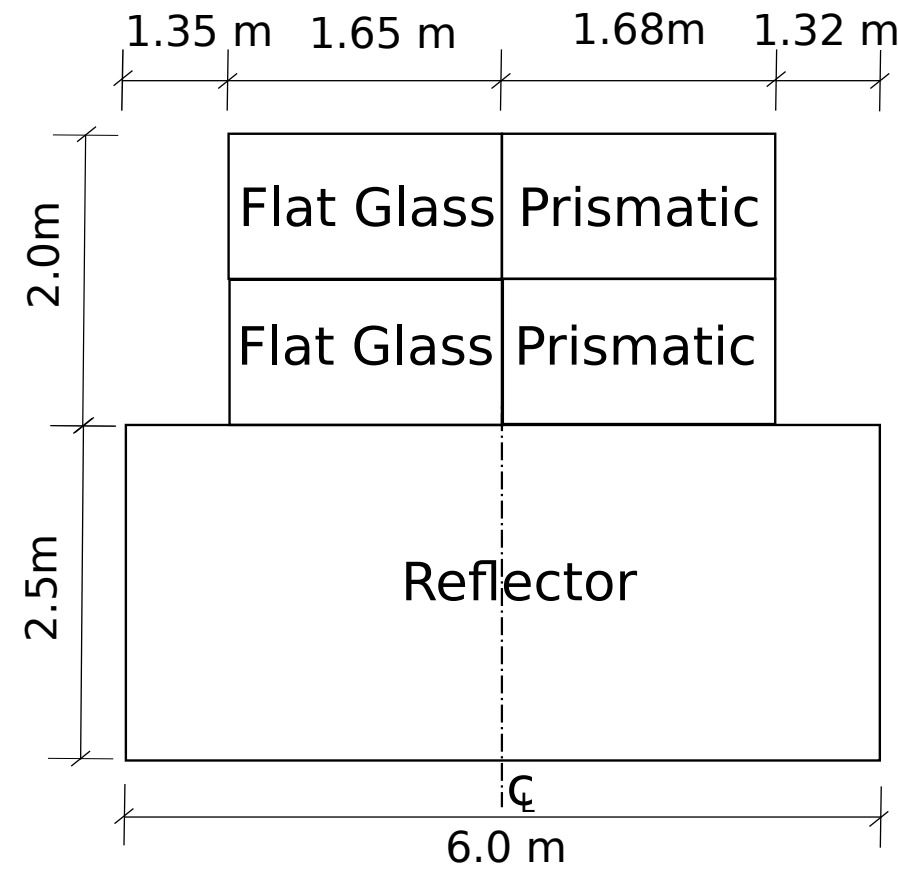

Fig. 1: Plan view of installed system

The tilt angles of the surfaces from horizontal were initially $\phi=20^{\circ}$ and $\omega=57^{\circ}$, see Figure D. After July, 2013 the reflector angle, $\phi$ was changed to $15^{\circ}$ to test the sensitivity of the proposed model to changes in input conditions. These values were chosen based on an initial optimization of the system using the methodology outlined in [20].

A set of control modules with no reflectors were installed at the same module tilt angle, and at a tilt angle of $30^{\circ}$ to closely match the optimal tilt angle for the region. This is slightly different than the overall optimal angle for the Kingston region of $35^{\circ}$ due to mechanical limitations of the racks used in the study. Based on analysis using the PVSYST modeling tool, the difference in irradiance on a surface at $30^{\circ}$ in the Kingston region is less than $0.4 \%$.

Meteorological measurements were made with two CMP 22 pyranometers, which measured global horizontal and diffuse horizontal irradiation, and temperature and wind speed measurements were taken at the site. The modules were monitored 
for short-circuit current $\left(I_{s c}\right)$ and for temperature at the top and bottom of the module.

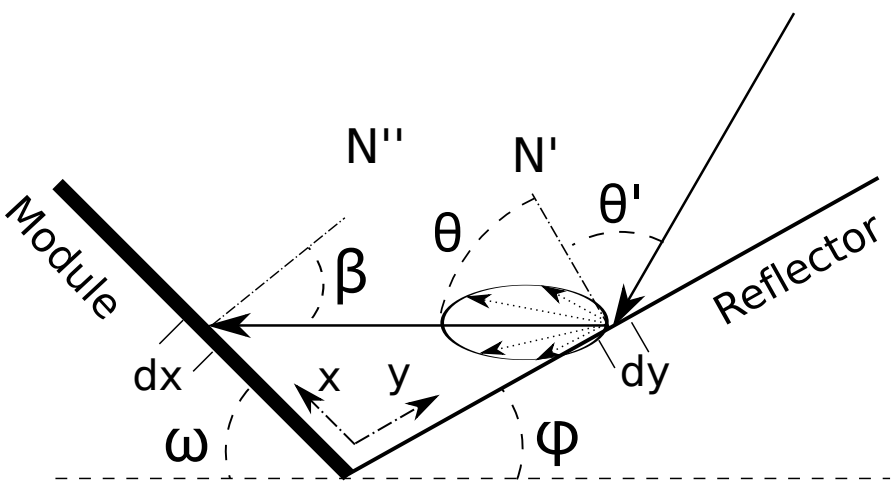

Fig. 2: Schematic of the modeled domain, all dimensions are in the plane normal to the surface which they are measuring.

The concentration system was installed in November 2011, and had two forms of reflector installed: i) semi-diffuse, flexible reflector made of an alumized PET laminate, (Foylon), and used until July, 2012, and ii) a specular aluminzed PET reflector (mylar), which was installed for the remainder of the test period. The Mylar and Foylon reflectors had a hemispherical reflectivity of approximately $73 \%$ and $84 \%$, respectively on installation. After 2 years of exposure, the hemispherical reflectivity of the Mylar and Foylon decreased to $60 \%$ and $65 \%$, respectively. It should be noted that both these films do not display good long-term weathering characteristics and should not be used for long term system analysis or PV system augmentation. The modules and reflector were cleaned of any major soiling and organic depositions when they were observed.

\section{B. Scatterometry}

A J.A Wollam variable angle spectroscopic ellipsometer (VASE) was utilized to performed scatterometry on both reflector materials. The reflector samples were adhered to a standard glass slide, which was placed on the rotating sample mount of the VASE. Scattered irradiation was measured using the collimated receiver from angles of $-10^{\circ}$ to $10^{\circ}$ degrees from specular, and at wavelengths of $300 \mathrm{~nm}-1000 \mathrm{~nm}$ in increments of $100 \mathrm{~nm}$. The sample was rotated with respect to the light source, and scatterometry was measured at angles of incidence relative to the reflector surface of $75^{\circ}, 60^{\circ}, 30^{\circ}$, and $15^{\circ}$. The reflectivity at each wavelength was weighted by the quantum efficincy of a c-Si module, in order to derive a spectrally integrated reflectivity. The experimental results are shown normalized by the maximum reflectivity at each incidence angle in Fig B].

The data is presented in a normalized fashion, as the primary purpose of this dataset is the validation of the use of the normalized Beckmann distribution introduced in section ??, and the derivation of physically representative scattering coefficients. Because the ellipsometer used is highly

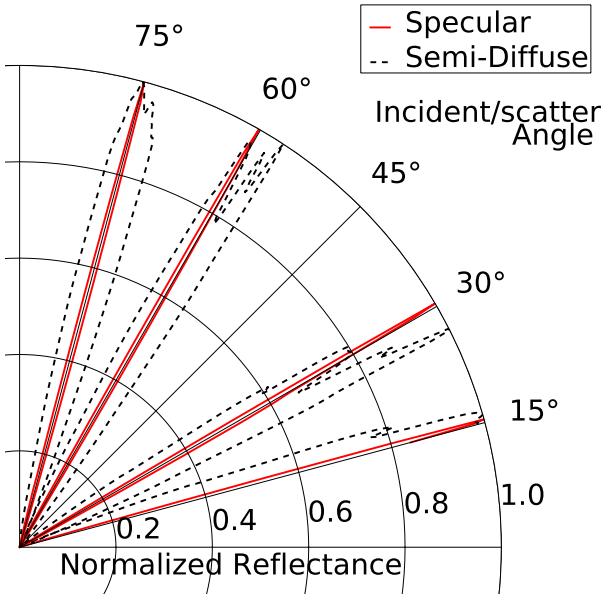

Fig. 3: Polar Co-ordinate view of normalized measured scatterometry data for the semi-diffuse and specular reflector.

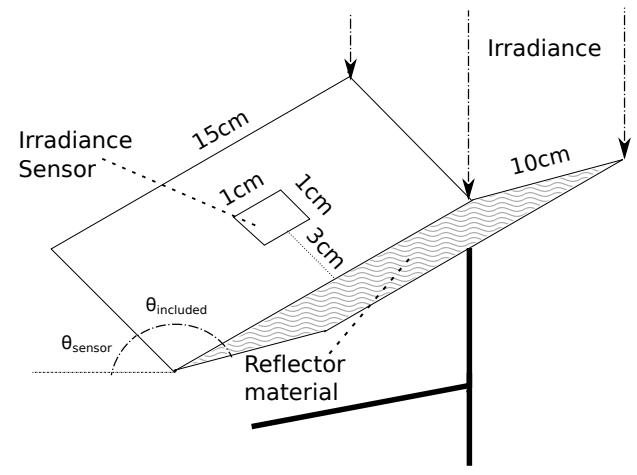

Fig. 4: Schematic depicting the apparatus utilized to simulate a reflector system under a solar simulator.

collimating, and scattering measurements were taken only in a two dimensional plane, the intensity measurements are not physically representative.

\section{Solar simulator testing}

A small-scale model of a large scale reflector system was constructed to produce validation data for the proposed reflector model under controlled laboratory conditions. A schematic of the test system utilized is shown in Figure 4 .

Irradiance was provided by a class AA solar simulator. The illuminated area projected onto the test apparatus had a visible diameter of $14 \mathrm{~cm}$, however there was significant attenuation at the edges of this projected area. Thus, it was ensured that at all times the sensor and the majority of the reflector were within an area described by a $9 \mathrm{~cm}$ diameter which maintained a uniform intensity within $3 \%$ of the peak area mean, as shown in Figure [5]

Tests were performed by setting an included angle between the reflector and sensor, $\left(\theta_{\text {included }}\right)$, and subsequently varying the sensor angle, $\left(\theta_{\text {sensor }}\right)$ from $0^{\circ}$ to $90^{\circ}$ from the horizontal. This process was repeated for multiple reflector samples, and a summary of the experimental results is shown in Figure 6 


\section{$20 \mathrm{~cm}$}

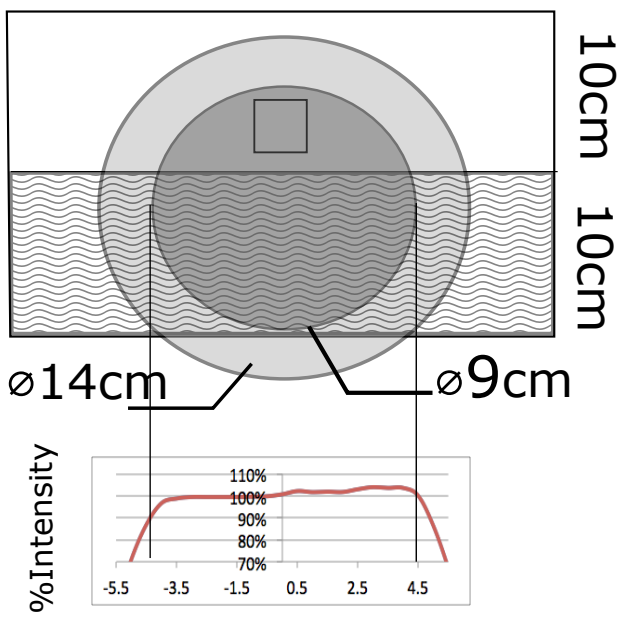

Distance from center $(\mathrm{cm})$

Fig. 5: Illustration of the illuminated area projected onto the surface of the test apparatus

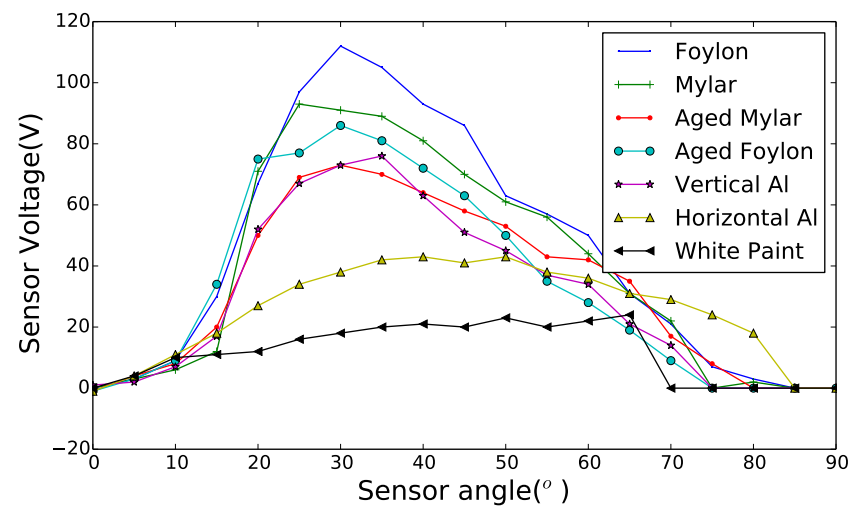

Fig. 6: Summary of reflector characteristics for a variety of materials, with an included angle $\left(\theta_{\text {included }}\right)$ of $100^{\circ}$. The sudden rise in output around $15^{\circ}$ is due to the top edge of the reflector image reaching the bottom edge of the sensor. The gradual decline in intensity after the peak is due to the dilution of the reflector image as is extends beyond the top of the sensor element, and is also dominated by angle of incidence effects.

\section{ModelLiNG}

A reflectance model based on a Bi-Directional Reflectance Function, BDRF, for an isotropic roughened surface is developed here to predict the performance of the reflector system. The model methodology presented in this paper uses the concepts of the BDRF of non-ideal surfaces rather than traditional geometric optics [24], [25]. This methodology allows for the evaluation of non-specular reflectors in planar concentration systems, which has been shown to increase the energy yields from these systems compared to purely specular reflectors. [5], [26]

The BDRF classifies the three dimensional scattering of light from a surface, and is described theoretically by Beckman and Spizzichino [27]. Recently, BDRF modelling and research has been a focus in the field of computer graphics [24], [28]-[30]. A modified version of the Cook-Torrance model is used in this paper, which is a commonly implemented model capable of simulating nearly specular to highly diffuse and anisotropic surfaces [24], [25]. Here, this BRDF formulation has been modified to ensure energy conservation.

The BDRF defines irradiance that reaches a module as a function of both the angle of incidence $\left(\theta^{\prime}\right)$ and the angle of viewing $(\theta)$ of the light. In the case of a perfectly specular reflector, the BDRF resembles the Dirac function, with a value of 1 when the incident angle equals the outgoing angle $\left(\theta=\theta^{\prime}\right)$, and 0 at any other viewing angle. This is the assumption of a ray-tracing concentrator model. However, real surfaces are not perfect specular reflectors and thus reflect light in a distribution as defined by the BDRF of the material, and is a combination of both diffuse and specular reflections.

\section{A. Model Domain}

The domain being considered in this model is shown as a function of the representative angles in Fig 2 . An integrative approach is taken in the analysis, where the contribution of irradiation to a differential point on the PV module ( $\mathrm{dx}$ ) from each differential scattering element on the reflector (dy) is computed. Thus, an integration is performed along the two principle directions of the array, $\mathrm{x}$ and $\mathrm{y}$ as shown in Figure $\mathbf{2}$.

In order to account for the diode topology of a PV module, the model divides each module domain into four distinct sections along the $\mathrm{x}$-coordinate. The minimum irradiance from any of the four domains is taken as the limiting energy incident on the module, and is used to predict the overall energy production of the module.

\section{B. Model Derivation}

A set of simplifying assumptions are used:

1) The reflector is assumed to be infinite and homogeneous along its length. Therefore reflection is only considered in a two dimensional plane normal to the infinite dimension. NOTE: For the experimental validation of the model, azimuthal effects of the finite reflector were taken into account as described in section III-C.

2) The reflector is a broadband reflector, and spectral attenuation is not taken into account.

The major implication of simplifying the domain of the reflector onto two-dimensional geometry is to properly account for the projection of the three-dimensional incident light ray onto the two dimensional domain. This is done using a modified version of the profile angle, $\alpha_{p}$ equation introduced in [3I] and recommended in [32]:

$$
\begin{array}{r}
\alpha_{s}=\pi / 2-\text { Zenith } \\
\tan \left(\alpha_{p}\right)=\frac{\tan \left(\alpha_{s}\right)}{\cos \left(\gamma_{\text {sun }}-\gamma_{\text {Array }}\right)} \\
G_{p}=G_{D N I} \frac{\sin \left(\alpha_{s}\right)}{\sin \left(\alpha_{p}\right)}
\end{array}
$$

Where Zenith is the solar zenith angle, $\alpha_{s}$ is the three dimensional solar elevation angle, $\gamma_{\text {sun }}$ and $\gamma_{\text {array }}$ are the 
solar and array azimuth angles, respectively, and $\alpha_{p}$ is the two-dimensional solar profile angle.

Recalling the model domain that is depicted in Fig 口, Equation 1 s shows the radiant intensity [W] that impacts the plane reflector at point dy and to a depth dz. Equation [5] represents the value of reflected radiant intensity per unit depth through a differential angle $[\mathrm{W} / \theta]$. Note that the BDRF is normalized such that $\int_{0}^{\pi} B D R F d \theta=1$. Equation 6 shows the radiant intensity per unit depth that strikes the surface of the module. Note that $\theta$ is defined in Equation $\square$ in terms of the angle of incidence of the differential ray onto the surface of the module, $\beta$, and the distance the ray has travelled $(r)$.

$$
\begin{aligned}
E_{r}^{i} & =E_{p} \cos \left(\theta^{\prime}\right) d y d z[W] \\
E_{r e f l} & \left.=E_{r}^{i} B D R F\left(\theta, \theta^{\prime}\right) \rho[W / \theta)\right] \\
E_{m} & =E_{r e f l} d \theta[W] \\
d \theta & =\frac{d y \cos (\beta)}{r} \\
\operatorname{BDRF}\left(\theta, \theta^{\prime}\right) & =\frac{D\left(\theta, \theta^{\prime}\right)}{\int_{-\frac{\pi}{2}}^{\frac{\pi}{2}} D\left(\theta, \theta^{\prime}\right) d \theta}
\end{aligned}
$$

Equations $4-8$ can be combined and integrated along the characteristic dimensions, and divided by the area of the module being analyzed $\left(A_{\text {module }}\right)$ to obtain the irradiance on the module surface.

$$
G_{m}^{i}=\frac{G_{p} \rho}{A_{m}} \int_{0}^{L_{\text {sect }}} \int_{L_{m, \min }}^{L_{m, \max }} B D R F\left(\theta, \theta^{\prime}\right) \cos \left(\theta^{\prime}\right) \frac{\cos (\beta)}{r} d x d y
$$

where $G_{m}^{i}$ is the irradiance $\left(W / m^{2}\right)$ on the surface of the module, $\rho$ is the specularly constant surface reflectivity, $\theta^{\prime}$ is constant for a given time step, and $\theta, \beta$, and $r$ are a function of the linear distances along reflector and module, $\mathrm{x}$ and $\mathrm{y}$ :

$$
\begin{aligned}
r & =\sqrt{\left(x^{2}+y^{2}-2 x y \cos (\phi+\omega-\pi / 2)\right.} \\
\theta & =\pi-\sin ^{-1}\left[\frac{r^{2}+y^{2}-x^{2}}{2 r y}\right] \\
\beta & =(\pi-\phi-\omega)-\theta
\end{aligned}
$$

and $\mathrm{D}\left(\theta, \theta^{\prime}\right)$ is given by the Beckmann distribution [27].

$$
\begin{aligned}
D\left(\theta, \theta^{\prime}\right) & =\frac{1}{m^{2} \cos ^{4}(\epsilon)} \exp ^{\frac{\cos ^{2}(\epsilon)-1}{m^{2} \cos ^{2}(\epsilon)}} \\
\epsilon & =\theta-\frac{\theta+\theta^{\prime}}{2}
\end{aligned}
$$

where $m$ is a physical parameter that represents the rms slope of surface roughness on the reflecting surface and generally $m \in\left[\begin{array}{ll}0 & 0.5\end{array}\right] . \epsilon$ is the angle from the surface normal of the vector bisecting $\theta$ and $\theta^{\prime}$.

The BDRF of the two surfaces used in this study were measured using the scatterometry techniques described in section [I-B]. The theoretical approximations for the BDRF using Equation [13] were also calculated for values of $\mathrm{m}=0.03$ for the specular reflector and $\mathrm{m}=0.07$ for the semi-diffuse reflector, which were found to be the closest match to the

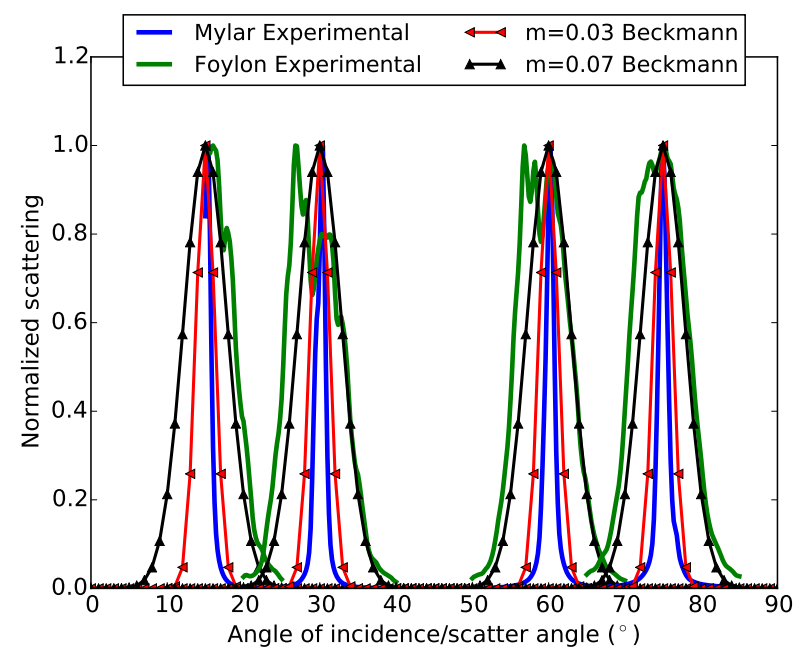

Fig. 7: Comparison of measured scatterometry results from both reflector materials to modelled BDRF distributions for two values of the roughness coefficient (m).

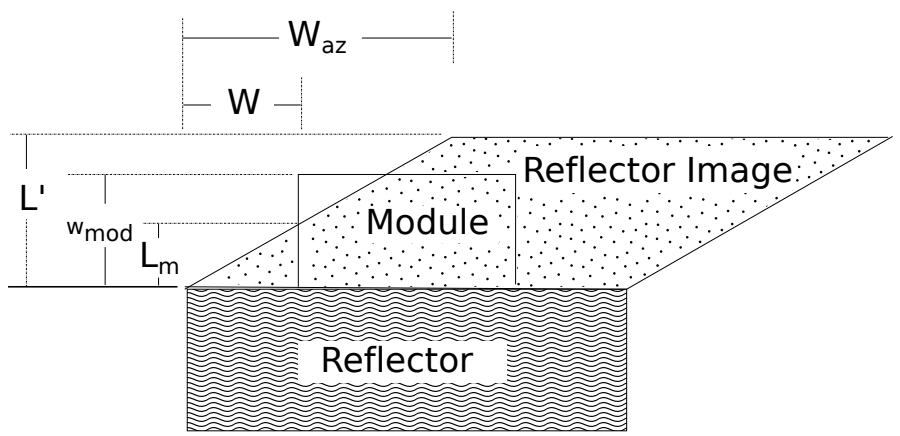

Fig. 8: Illustration showing potential partial illumination of a module by a finite reflector length. South is down.

measured data, and the comparison of the experimental and theoretical values is shown in Fig $\square$

The diffuse contribution of irradiation was evaluated using the diffuse view factor between the reflector and PV module, $F_{r m}$, as described by [33].

$$
F_{r m}=\frac{R+1-\left(R^{2}+1-2 \times R \times \cos \left(\omega_{\text {incl }}\right)\right)^{\frac{1}{2}}}{2}
$$

where $\mathrm{R}$ is the ratio of module length to reflector length, and $\omega_{i n c l}$ is the angle between the two surfaces.

\section{Azimuthal correction}

The 2D model proposed above was modified to account for the illumination effects of finite reflector lengths. It is possible that in the morning and evening, irradaince at high azimuth angles would not illuminate the entire module as shown in Figure 8 . This was accounted for by calculating the non-illuminated area of the module during these periods, and adjusting the expected energy output by the calculated area ratio. This is a process similar to the one implemented by Bollentin [177].

The equation proposed by Bollentin utilized was modified to the following: 


$$
\begin{aligned}
L^{\prime} & =\frac{\text { wrefl }}{\sin \left(2 \times \omega+\phi-\alpha_{p}\right) \times \sin \left(\alpha_{p}-\omega\right)} \\
W_{a z} & =-\tan (\gamma) *\left(L^{\prime} \times \cos (\omega)+w r e f l \times \cos (\phi)(17)\right. \\
L_{m} & =a b s\left(L^{\prime} / W_{a z} \times W\right) \\
A R & =1-\left|\frac{.5 \times\left(L^{\prime}-L_{m}\right) \times \frac{W_{a z}}{L^{\prime}} \times\left(L^{\prime}-L_{m}\right)}{L^{\prime} \times w \bmod }\right|
\end{aligned}
$$

where $L^{\prime}=\operatorname{Lmod}$ if $L^{\prime}>\operatorname{Lmod}$. The value $A R$ represents the effective area ratio of the area not illuminated by the specular image of the reflection. As a first order approximation, the total irradiance on the surface of the module is given by $G_{m}^{i} * A R$ in cases where edge effects are considered.

\section{Model Implementation}

The BDRF model was run iteratively for the full measured dataset of incoming irradiance and zenith angles, and the dual integration was run at each step. The amount of irradiation that directly impacts the module was evaluated using the Perez irradiation translation model [34]. Once the total inplane irradiation on the face of the module was evaluated, the predicted module output was calculated using the methodologies outlined in [35] using coefficients derived from the control module. Note that only $I_{s c}$ was collected from the modules, and therefore the validation of the model was performed using $I_{s c}$ rather than power. $I_{s c}$ is an excellent predictor of effective irradaince on the plane of the array [35], and is therefore well suited for validating the model. However, when comparing the annual outputs from the modules, the collected $I_{s c}$ is utilized to calculate effective irradiance, $E_{e}$ in the Sandia Array Performance Model [36], and used with the collected cell temperature to estimate the power production from each module.

\section{E. Thermal model}

The Sandia cell temperature model [36] was modified to predict the temperature of modules under low concentration. Thus, the thermal model utilized for this study is :

$$
T_{\text {Cell }}=G_{D N I} \times C_{0}+G_{d i f f} \times C_{1}+\exp \left(C_{2} \times G_{D N I}\right)+T_{a}
$$

Coefficients were obtained using a least-squares optimization and were found to be $C_{0}=0.0232, C_{1}=0.0276, C_{2}=-$ 0.00011. These coefficients gave a Normalized Root Mean Squared Error (NRMSE) of $6 \%$ and a Mean Bias Error (MBE) of $0.1 \%$. A correlation plot of this fit is shown in Figure $Q$

Interestingly, the measured data display temperature spikes for short periods during the day, where the cell to which the thermocouple was attached could reach temperatures approaching $100^{\circ} \mathrm{C}$ for a short period of time, as seen in Figure [0. One possible explanation is that these spikes are due to inconsistent illumination from the reflector, however the reflector is relatively uniform, and another possible explanation is that these temperature spikes are due to the normal "patchwork" appearance of cell temperatures for a short-circuited module, as shown in Figure 피.

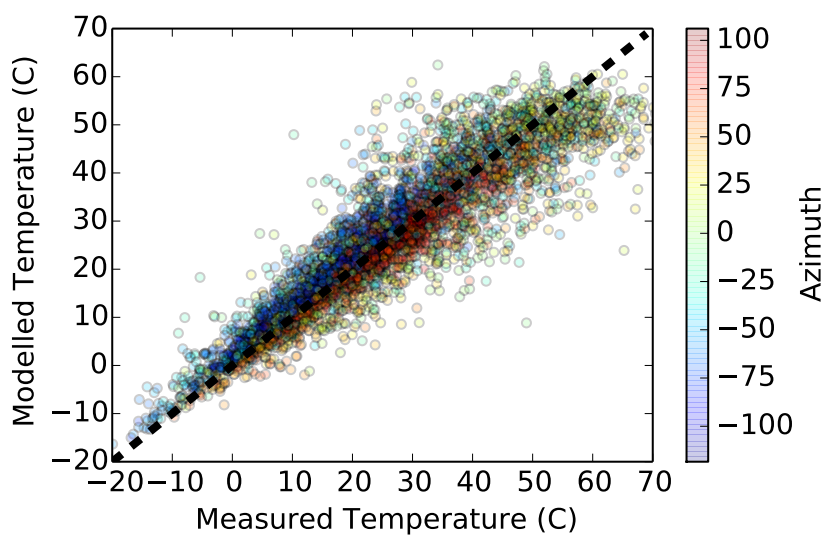

Fig. 9: Correlation plot showing the quality of the fit of the temperature model.

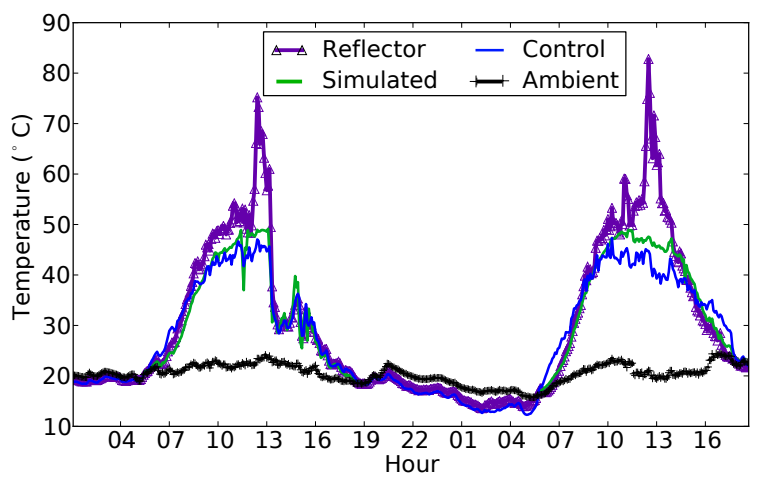

Fig. 10: Thermal variations for May 29 and May 30, showing the thermal spike effect, and the fit of the proposed thermal model.

\section{F. Model Validation}

Initial validations were performed as comparisons to the solar simulator measurements described in section II-C. It was noted that the model accurately predicted the characteristics of a new Mylar $(\mathrm{m}=0.03$, rho $=0.73)$ and Foylon $(\mathrm{m}=0.07$, rho $=0.84$ ) reflector as seen in Figure [12 and Figure [13]. In addition, the imaging of the reflection onto the sensor is properly handled, as demonstrated by the matched uptake at $5^{\circ}$ and $15^{\circ}$ for $\theta_{\text {included }}=100^{\circ}$ and $\theta_{\text {included }}=90^{\circ}$, in Figures [2] and [3] respectively.

The model was then applied to the entire dataset, the model predictions are in reasonable accord with the experimental data, as shown in Figure 14. The prismatic glass module was fit with an NRMSE of $12 \%$ and an MBE of $1 \%$, and the flat glass module was fit with an NRMSE of 14\% and an MBE of $2 \%$. Figure 15 presents a time series showing a typical fit of hourly data . It is important to note that beyond the tuning of the temperature model, no empirical parameters are utilized. All user inputs to the model were based on the physical properties of the system. 


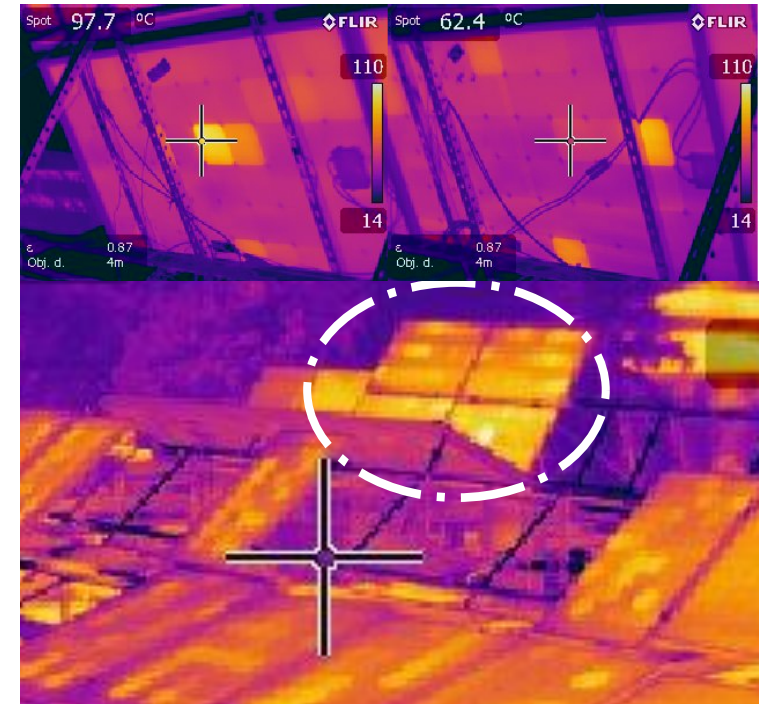

Fig. 11: Top:Two Infrared photographs of a module backsheet, with reflector augmentation taken 1 minute apart. A moving patchwork of hotspot cells is apparent. Bottom: view of multiple short-circuited modules. Only the modules highlighted with the white circle have reflector augmentation.

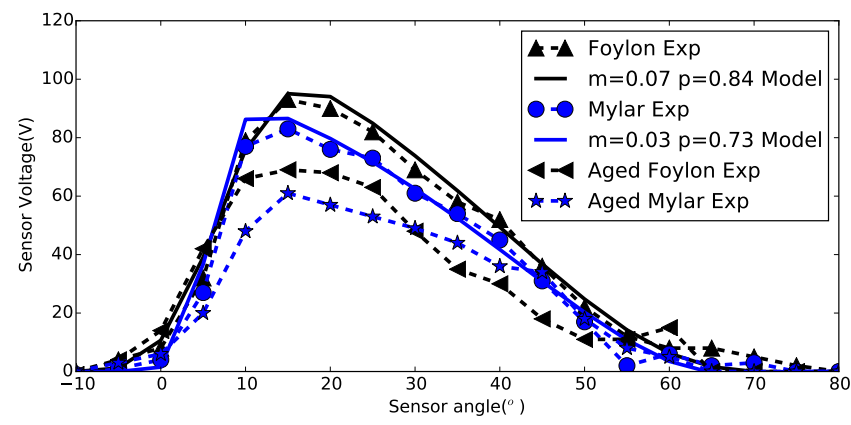

Fig. 12: Comparison of experimental and modelled results for an included angle $\left(\theta_{\text {included }}\right)$ of $100^{\circ}$. Also shown is the effects of approximately 1.5 years of weathering on the optical performance of the reflectors.

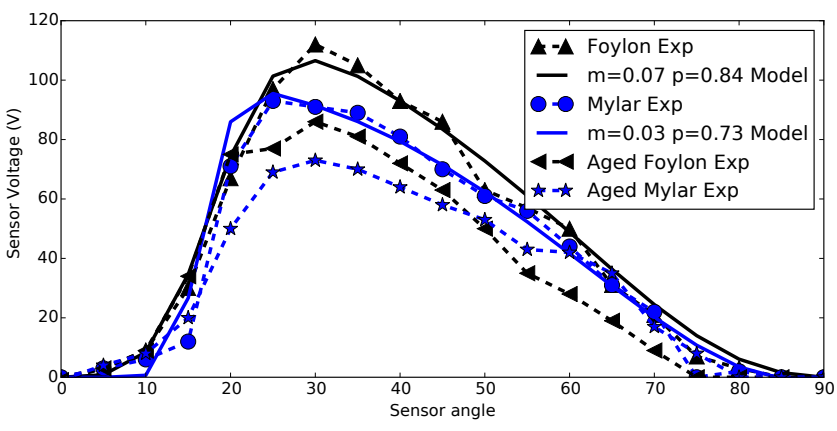

Fig. 13: Comparison of experimental and modelled results for an included angle $\left(\theta_{\text {included }}\right)$ of $90^{\circ}$. Also shown is the effects of approximately 1.5 years of weathering on the optical performance of the reflectors.

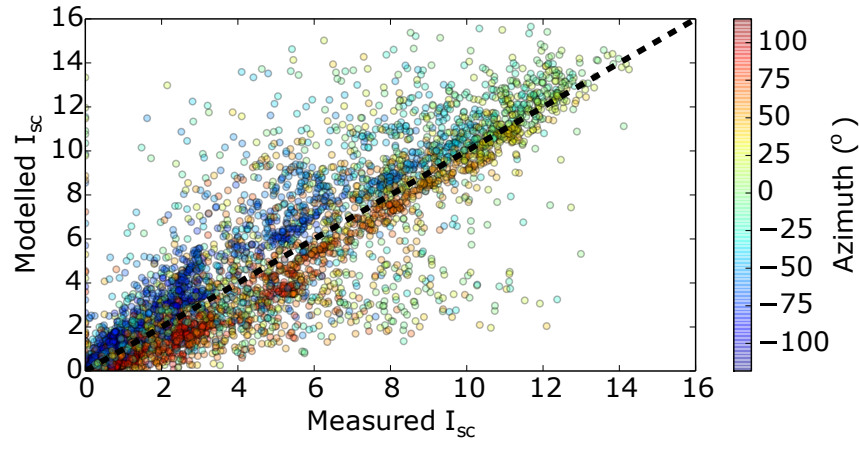

Fig. 14: Correlation plot showing the fit of hourly data.

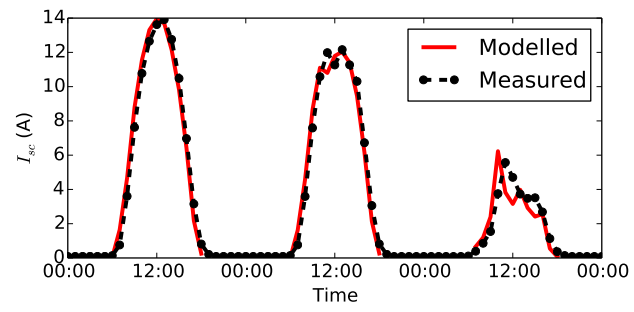

Fig. 15: Three typical days from April $7^{\text {th }}-9^{\text {th }}$ demonstrating the model fit to experimental data.

\section{EXPERIMENTAL RESULTS}

Fig 10 presents the weekly normalized increase in $P_{m p}$ between reflector and control modules. On average, the use of a non-tracking planar reflector can increase system performance for a module at the same angle (as characterized by $\int P_{m p}$ ) by $45 \%$ for a traditional flat glass module and by $40 \%$ for a prismatic glass module.

It should be noted that the high module angle of $57^{\circ}$ is not the optimal non-augmented orientation for this latitude. An identical control module was mounted at $30^{\circ}$ for the same period of testing, which is within $0.4 \%$ of the yearly energy yield of to the regional optimal angle of $35^{\circ}$. A Comparison of the energy output of the reflector augmented modules to this optimal control module gives an the energy performance increase of $18 \%$ for both modules.

The effects of the reflector are also characterized in Figure [7], indicating the dependence of output ratio on solar zenith angle over the year for non-cloudy days for all modules.

Fig 18 displays a probability density function for module temperatures. It can be seen that for the majority of operation, the module operates below or near to Normal Operating Cell Temperature (NOCT), $48^{\circ} \mathrm{C}$. There are some occasions where the cell temperature rises above $90^{\circ} \mathrm{C}$, which is beyond the maximum design temperature of some commercial modules. The results of this study show that it is in the best interests of module manufacturers to ensure that segments of their modules can withstand elevated temperature operation in order to take advantage of the benefits of low-concentration system integration. 


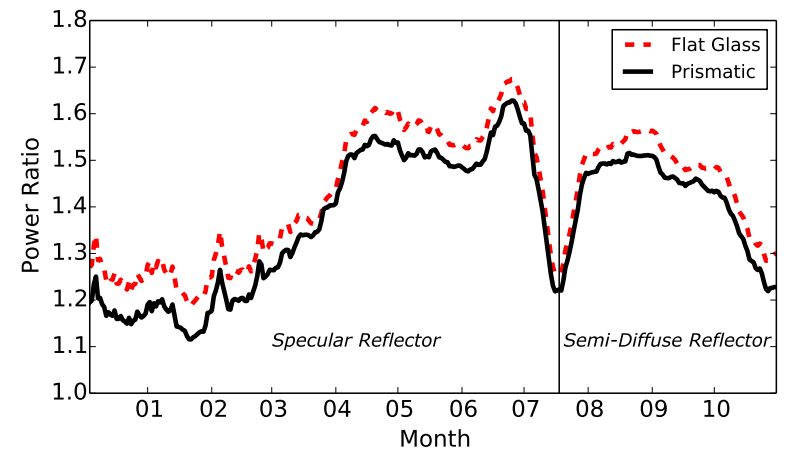

Fig. 16: Time series of daily power boost due to reflectors as compared to control modules, averaged over a one week period. The decrease in performance in July represents the time when the reflectors were reduced to horizontal in order to change the reflective material.

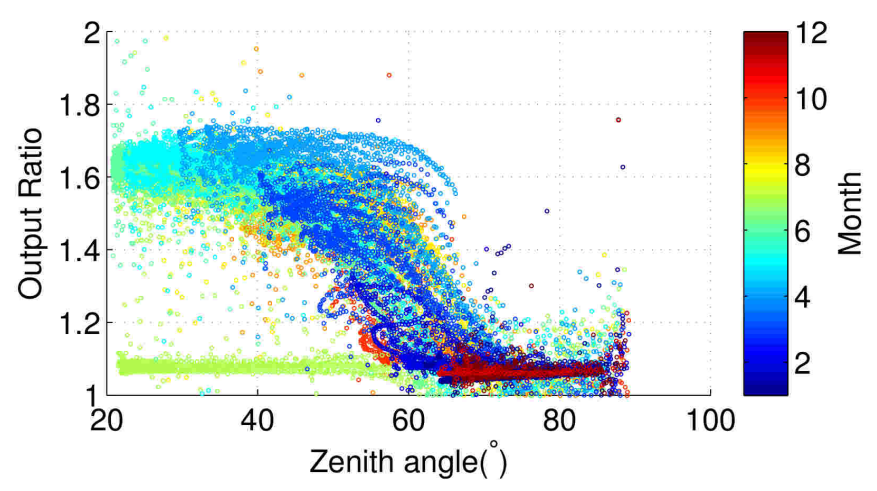

(a) Flat Glass

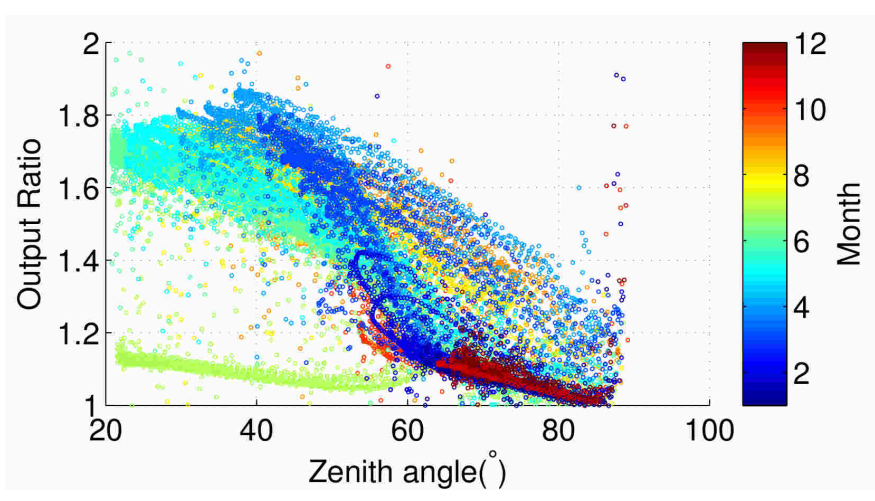

(b) Prismatic Glass

Fig. 17: Clear day output ratios (ratio of reflector augmented to control module) as a function of zenith angle for one year of data

\section{Model SEnsitivity ANALYSIS}

An optimization was performed to determine the best topology layout for the two typical reflector surfaces investigated in this study. It was assumed that the length of the module remained constant at $1 \mathrm{~m}$, due to manufacturing limits. The length of the reflector was also kept constant at $2.5 \mathrm{~m}$, it is assumed that this length of reflector could be reduced through optimization, however a large reflector which will not

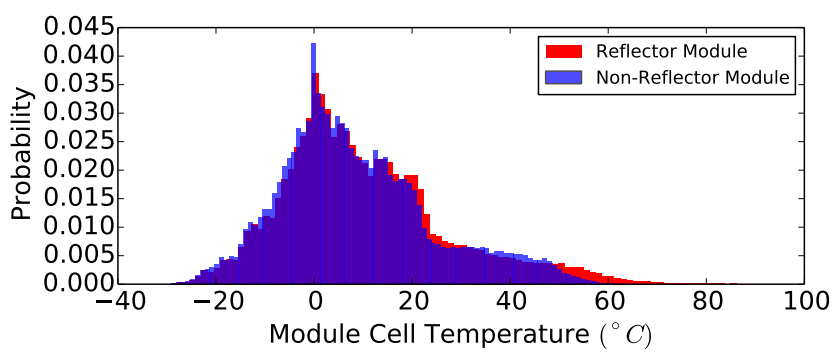

Fig. 18: A probability distribution plot of cell temperatures, showing a slight increase in cell temperatures for reflector augmented moduels.

introduce edge effects, was chosen for this optimization, and it is assumed that the output would not be largely affected by changes in reflector length around this ideal. Therefore, only panel angle $(\omega)$, reflector angle $(\phi)$ and the scattering coefficient $(m)$ were optimized for a location in Kingston, Ontario, Canada (44. $\left.140 \mathrm{~N}, 76^{\circ} 300 \mathrm{~W}\right)$.

Combinations of $\omega, \phi$, and $m$ were calculated within the domain:

$$
\begin{array}{r}
\phi \in[0,10 \ldots 60] \\
\omega \in[25,35 \ldots 85] \\
m \in[0.01,0.04,0.08,0.15,0.65] \\
\rho=0.8
\end{array}
$$

Note that the exact experimental apparatus is not included in this domain $\left(\phi=20^{\circ}\right.$ and $\left.15^{\circ}, \omega=57^{\circ}\right)$, however the intention of this analysis is to sample the entire domain at regular intervals rather than to replicate the exact experimental apparatus, as was done in Section 피-E.

The total boost, or increase in performance relative to a non-augmented module placed at the optimal orientation for the Kingston site $\left(35^{\circ}\right)$ was calculated for each case, and the results are shown in Figure [1. From this sensitivity analysis, it can be seen that the maximum theoretical increase in performance available is $30 \%$ over a non-augmented system. This is true for a variety of systems with an integrated reflectivity of 0.8 , a scattering coefficient up to 0.08 and for a reflector angles between $30^{\circ}$ and $40^{\circ}$.

It can be seen that there is a relatively low sensitivity to the overall module boost near the optimal point for each graph. However, the daily and yearly distributions of irradiance can vary by a large degree between different settings, which may be useful for load matching. Figures [20- 22 show the effects of varying $\phi, \omega$, and $m$ from a base case of $\phi=20^{\circ}, \omega=55^{\circ}$ and $m=0.01$

In order to identify if this form of system can be economically feasible, a rough economic case is presented. In this system, the simulated reflector length was approximately twice the length of the modules being simulated, thus 2:1 area ratio of reflector to module surface area is assumed. At a 2:1 area ratio, the distance between rows of PV modules would not be largely affected as compared to a non-reflector system. Thus, it is assumed that land costs will not make a 


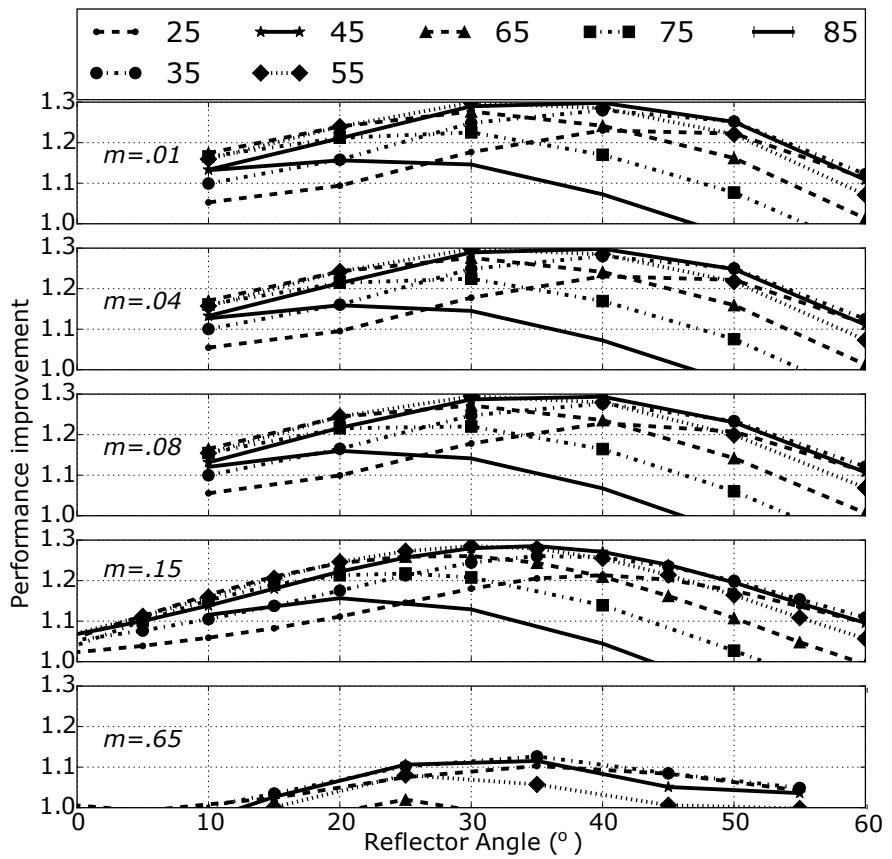

Fig. 19: Results of evaluation of the reflector code the simulation domain. The legend indicates the module angle $(\omega)$ in degrees, and each graph represents a different value of the scattering coefficient, $\mathrm{m}$.

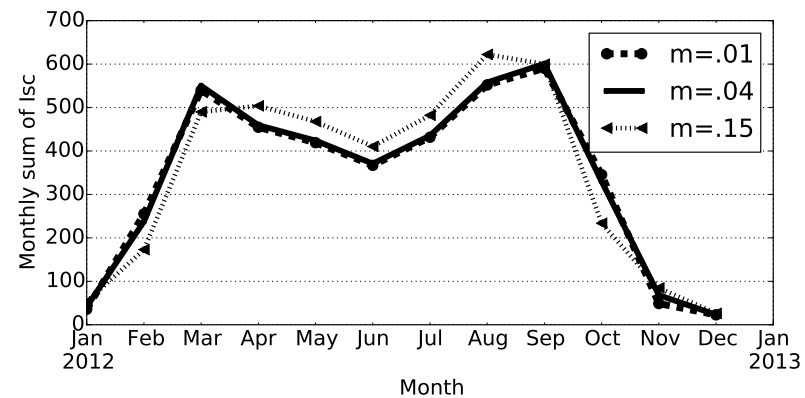

Fig. 20: Effects on Isc when varying the scattering coefficient, $\mathrm{m}$, keeping $\omega=55^{\circ}$ and $\phi=20^{\circ}$

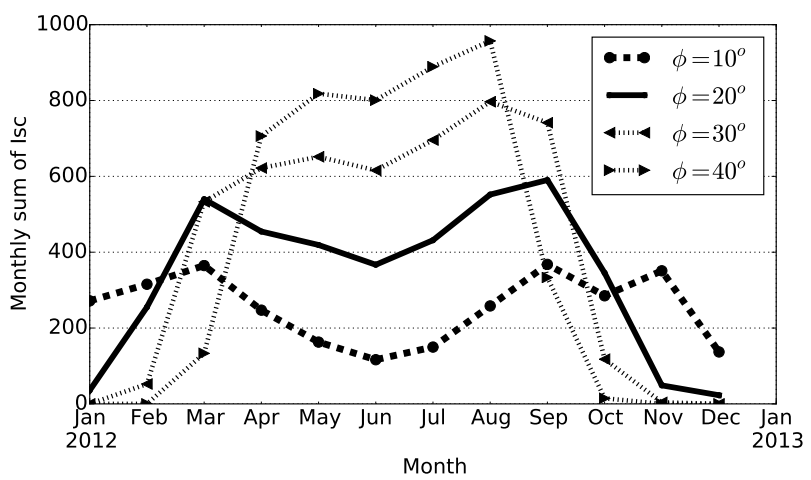

Fig. 21: Effects on Isc when varying $\phi$, keeping $\omega=55^{\circ}$ and $m=0.01$.

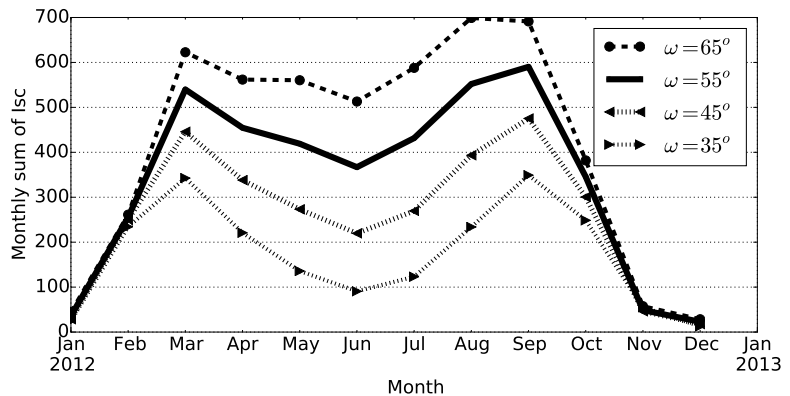

Fig. 22: Effects on Isc when varying $\omega$ keeping $\phi=20^{\circ}$ and $m=0.01$.

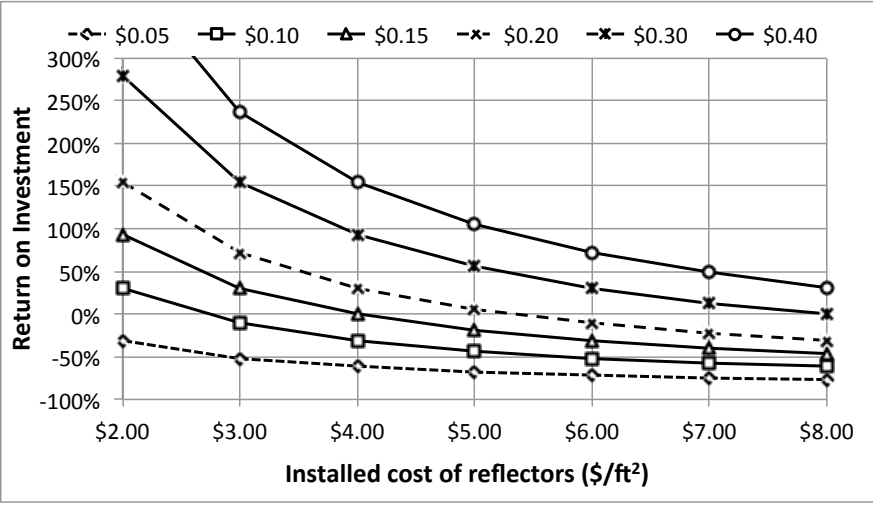

Fig. 23: Economic sensitivity analysis, showing the return on investment of the installation of a reflector system, as the cost of the reflector and system PPA rate are varied. This assumes an $8 \%$ cost of capital and 20 year project lifetime.

large difference in the economics of the project, and are not included in this analysis. The modules utilized in this system are $1 \mathrm{~m} \times 1.65 \mathrm{~m}$, or $1.65 \mathrm{~m}^{2}$, thus the equivalent reflector area is $3.3 \mathrm{~m}^{2}$. In a simple economic case, we will assume an interest rate of $8 \%$ over 20 years for the capital expense of the reflector system. The variables of interest are the installed cost of the reflector system, expressed in $\$ / f t^{2}$, and the Power Purchase Agreement (PPA) rate received from the PV asset. From the results shown in Figure 23, the return on investment of installing a reflector system is highly dependent on the installed costs of the reflector. Further investigation is required to determine appropriate cost models for this component.

It should be noted that these estimations do not necessarily represent the overall economically optimal arrangement for a practical system where a DC overrate of $20 \%-30 \%$ is common. The optimal arrangement of reflectors will tend to increase the system output around periods of the day that already experience high levels of irradiance. Thus, much of the additional energy available from the reflector mirrors may not be utilized effectively by the inverters. Therefore, future work should investigate the optimal arrangement of a reflector system to ensure maximum inverter utilization. 


\section{FUTURE WORK}

As mentioned previously, future work should investigate the effects of DC overrate on the optimal arrangement of a reflector system. It is conceivable that the sytem could be optimized to maximize total energy yield, or could be optimized to produce more energy in the mornings and evenings in order to provide passive load balancing.

Another interesting extension of this work is to perform an optimization on the shape of the surface BRDF. Though the model used in this research represents a symmetrical BRDF, micro-engineered surfaces could be used to provide other non-symmetric BRDF profiles which could increase the effective output of planar concentrator systems. In addition this model should be adapted for solar thermal systems and photovoltaic solar thermal (PVT) systems, as the increased operating temperatures would increase the exergy [37]-[39] of both and decrease the spike annealing time of the latter [40], [41], increasing yields further.

Finally, an extension of the presented economic analysis is warranted to determine if the additional capital costs of the reflector system are warranted for both existing solar farms and retrofits.

\section{CONCLUSIONS}

This analysis has demonstrated the use of a physically based BRDF model for evaluating the use of planar reflectors in low-concentration PV systems. The model provides a good fit to experimental data with an MBE of $1 \%-2 \%$ and an hourly NRMSE of $12 \%-14 \%$.

From this analysis it can be seen that the use of non-tracking planar concentrators are a low cost method of increasing the performance of traditional PV systems. Over a year of outdoors testing, the system has been shown to improve energy yield (as characterized by $\int P_{m p}$ ) by $\mathbf{4 5 \%}$ for a traditional flat glass module and by $\mathbf{4 0 \%}$ for a prismatic glass module. Compared to a module oriented at the local non-augmented optimal angle, the experimental boost was seen to be $18 \%$. A sensitivity analysis shows that at this latitude a maximum increase of $30 \%$ is possible, with a reflector material having a hemispherical reflectance $(\rho)$ of $80 \%$.

In the future this modeling methodology can be used to optimize the reflector topology and identify the potential for increased energy harvest from both existing PV systems and newly built PV arrays.

\section{ACKNOWLEDGEMENTS}

The authors would like to acknowledge the work of $\mathrm{H}$. McLaren, J. Fairborn, Q. Bentley, D. Carter and A. Babasola and the support of the Sustainable Energy Applied Research Centre at St. Lawrence College, and to the forward-looking industry partners of this project who made it possible. This project was supported by the Natural Sciences and Engineering Research Council of Canada and a Social Sciences and Humanities Research Council of Canada grant.

\section{REFERENCES}

[1] J. M. Pearce, "Photovoltaics - a path to sustainable futures," Futures, vol. 34, no. 7, pp. 663-674, Sep. 2002.

[2] K. Branker, M. Pathak, and J. Pearce, "A review of solar photovoltaic levelized cost of electricity," Renewable and Sustainable Energy Reviews, vol. 15, no. 9, pp. 4470-4482, Dec. 2011.

[3] C. Deline, A. Dobos, S. Janzou, J. Meydbray, and M. Donovan, "A simplified model of uniform shading in large photovoltaic arrays," Solar Energy, vol. 96, no. 0, pp. 274 - 282, 2013.

[4] I. R. Cole and R. Gottschalg, "Modelling the efficiency of terrestrial photovoltaic systems," in Proceedings, Photovoltaic Science, Applications and Technology (PVSAT-6), University of Southampton, UK, 2010, this is a conference paper.

[5] M. Rnnelid and B. Karlsson, "The use of corugated booster reflectors for solar collector fields," Solar Energy, vol. 65, no. 6, pp. 343-351, Apr. 1999.

[6] H. Chiam, "Stationary reflector-augmented flat-plate collectors," Solar Energy, vol. 29, no. 1, pp. 65-69, 1982.

[7] D. Grimmer, K. Zinn, K. Herr, and B. Wood, "Augmented solar energy collection using different types of planar reflective surfaces; theoretical calculations and experimental results," Solar Energy, vol. 21, no. 6, pp. 497-501, 1978.

[8] D. McDaniels, D. Lowndes, H. Mathew, J. Reynolds, and R. Gray, "Enhanced solar energy collection using reflector-solar thermal collector combinations," Solar Energy, vol. 17, no. 5, pp. 277-283, Nov. 1975.

[9] S. C. Seitel, "Collector performance enhancement with flat reflectors," Solar Energy, vol. 17, no. 5, pp. 291-295, Nov. 1975.

[10] L. T. Kosti and Z. T. Pavlovi, "Optimal position of flat plate reflectors of solar thermal collector," Energy and Buildings, vol. 45, pp. 161-168, Feb. 2012.

[11] M. D. J. Pucar and A. R. Despic, "The enhancement of energy gain of solar collectors and photovoltaic panels by the reflection of solar beams," Energy, vol. 27, no. 3, pp. 205-223, Mar. 2002.

[12] B. Perers, B. Karlsson, and M. Bergkvist, "Intensity distribution in the collector plane from structured booster reflectors with rolling grooves and corrugations," Solar Energy, vol. 53, no. 2, pp. 215-226, Aug. 1994.

[13] M. Hall, A. Roos, and B. Karlsson, "Reflector materials for twodimensional low-concentrating photovoltaic systems: the effect of specular versus diffuse reflectance on the module efficiency," Progress in Photovoltaics: Research and Applications, vol. 13, no. 3, p. 217233, 2005.

[14] S. L. Grassie and N. R. Sheridan, "The use of planar reflectors for increasing the energy yield of flat-plate collectors," Solar Energy, vol. 19 , no. 6, pp. 663-668, 1977.

[15] H. Tanaka, "Solar thermal collector augmented by flat plate booster reflector: Optimum inclination of collector and reflector," Applied Energy, vol. 88, no. 4, pp. 1395-1404, Apr. 2011.

[16] H. M. S. Hussein, G. E. Ahmad, and M. A. Mohamad, "Optimization of operational and design parameters of plane reflector-tilted flat plate solar collector systems," Energy, vol. 25, no. 6, pp. 529-542, Jun. 2000 .

[17] J. W. Bollentin and R. D. Wilk, "Modeling the solar irradiation on flat plate collectors augmented with planar reflectors," Solar Energy, vol. 55, no. 5, pp. 343-354, Nov. 1995.

[18] B. Perers and B. Karlsson, "External reflectors for large solar collector arrays, simulation model and experimental results," Solar Energy, vol. 51, no. 5, pp. 327-337, 1993.

[19] T. Matsushima, T. Setaka, and S. Muroyama, "Concentrating solar module with horizontal reflectors," Solar Energy Materials and Solar Cells, vol. 75, no. 34, pp. 603-612, Feb. 2003.

[20] Rob W. Andrews, Nabeil Alazzam, and Joshua M. Pearce, "Model of loss mechanisms for low optical concentratioon on solar photovoltaic arrays with planar reflectors," in 40th American Solar Energy Society National Solar Conference Proceedings, Rayleigh NC, 2011, pp. 446453.

[21] J. Laird, "Facility puts solar products to test," Renewable Energy Focus, vol. 12, no. 5, pp. 20-22, Sep. 2011.

[22] R. Andrews, A. Pollard, and J. Pearce, "Photovoltaic system performance enhancement with non-tracking planar concentrators: Experimental results and bdrf based modelling," in Photovoltaic Specialists Conference (PVSC), 2013 IEEE 39th, June 2013, pp. 0229-0234. 
[23] J. M. Pearce, A. Babasola, and R. Andrews, "Open solar photovoltaic systems optimization," Proceedings of the 16th Annual National Collegiate Inventors and Innovators Alliance Conference, Open 2012, 2012.

[24] R. L. Cook and K. E. Torrance, "A reflectance model for computer graphics," ACM Trans. Graph., vol. 1, no. 1, p. 724, Jan. 1982.

[25] Christophe Schlick, "An inexpensive BRDF model for physcially-based rendering," Computer Graphics Forum, vol. 13, no. 3, pp. 233-246, 1994.

[26] M Hall, A Roos, and Bjorn Karlsson, "Reflector materials for twodimensional low-concentrating photovoltaic systems: the effect of specular versus diffuse reflectance efficiency on the module," Progress in Photovoltaics: Research and Applications, vol. 13, no. 3, pp. 217 233, 2005.

[27] P. Beckmann and A. Spizzichino, "The scattering of electromagnetic waves from rough surfaces," Norwood, MA, Artech House, Inc., 1987, 511 p., vol. 1, 1987.

[28] K. E. Torrance and E. M. Sparrow, "Theory for off-specular reflection from roughened surfaces," Journal of the Optical Society of America, vol. 57, no. 9, pp. 1105-1112, Sep. 1967.

[29] X. D. He, K. E. Torrance, F. X. Sillion, and D. P. Greenberg, "A comprehensive physical model for light reflection," SIGGRAPH Comput. Graph., vol. 25, no. 4, p. 175186, Jul. 1991.

[30] K. J. Dana, B. van Ginneken, S. K. Nayar, and J. J. Koenderink, "Reflectance and texture of real-world surfaces," ACM Trans. Graph. vol. 18, no. 1, p. 134, Jan. 1999.

[31] J. A. Duffie and W. A. Beckman, Solar Engineering of Thermal Processes, 2nd ed. Wiley-Interscience, Oct. 1991.

[32] B. Perers and B. Karlsson, "External reflectors for large solar collector arrays, simulation model and experimental results," Solar Energy, vol. 51, no. 5, pp. 327-337, Jan. 1993.

[33] P. Schrder and P. Hanrahan, "On the form factor between two polygons," in Proceedings of the 20th annual conference on Computer graphics and interactive techniques, ser. SIGGRAPH '93. New York, NY, USA: ACM, 1993, p. 163164.

[34] R. Perez, P. Ineichen, R. Seals, J. Michalsky, and R. Stewart, "Modeling daylight availability and irradiance components from direct and global irradiance," Solar Energy, vol. 44, no. 5, pp. 271-289, 1990.

[35] R. W. Andrews, A. Pollard, and J. M. Pearce, "Improved parametric empirical determination of module short circuit current for modelling and optimization of solar photovoltaic systems," Solar Energy, vol. 86, no. 9, pp. 2240-2254, Sep. 2012

[36] D. L. King, W. E. Boyson, and J. A. Kratochvil, "Photovoltaic array performance model," Sandia, vol. SAND2004-3535, Aug. 2004

[37] W. G. J. van Helden, R. J. C. van Zolingen, and H. A. Zondag, "Pv thermal systems: Pv panels supplying renewable electricity and heat," Progress in Photovoltaics: Research and Applications, vol. 12, no. 6, pp. 415-426, 2004.

[38] N. Lior and N. Zhang, "Energy, exergy, and second law performance criteria," Energy, vol. 32, no. 4, pp. $281-296,2007$, \{ECOS $\}$ 05. 18th International Conference on Efficiency, Cost, Optimization, Simulation, and Environmental Impact of Energy Systems $\{$ ECOS $\} 05$.

[39] H. Zondag, "Flat-plate pv-thermal collectors and systems: A review," Renewable and Sustainable Energy Reviews, vol. 12, no. 4, pp. 891 959, 2008.

[40] M. Pathak, P. Sanders, and J. Pearce, "Optimizing limited solar roof access by exergy analysis of solar thermal, photovoltaic, and hybrid photovoltaic thermal systems," Applied Energy, vol. 120, no. 0, pp. 115 $-124,2014$.

[41] M. Pathak, J. Pearce, and S. Harrison, "Effects on amorphous silicon photovoltaic performance from high-temperature annealing pulses in photovoltaic thermal hybrid devices," Solar Energy Materials and Solar Cells, vol. 100, no. 0, pp. 199 - 203, 2012, photovoltaics, Solar Energy Materials, and Technologies: Cancun 2010. 